\title{
Associations between quality of life and central auditory processing in seniors: preliminary results
}

A Amaral ${ }^{1}$ C Silva ${ }^{1}$ A Alves ${ }^{1}$ C Prata ${ }^{1}$ C Fernandes ${ }^{1}$ P Simões ${ }^{1}$ M Nascimento ${ }^{1}$, C Rocha ${ }^{1}$, on behalf of the AGA@4life Consortium ${ }^{1}$ ESTeSC - Coimbra Health School, Instituto Politécnico de Coimbra, Portugal

Introduction:

Several health-related conditions have been reported to be important for quality of life (QoL) (Raggi et al., 2016). Central auditory processing (CAP) corresponds to the perceptual rocessing of the auditory information in the central nervous system (Musiek \& Baran, 2007). With aging, alterations at the level of perception and detection of the sound in the central and peripheral system occur and have effects on the senior's QoL.

Objectives:

To analyse associations between CAP and QoL, considering sociodemographic variables.

Methods:

This study was conducted in a cross-sectional and descriptive design. Ethical requirements are respected. Participants: 19 seniors with average age of $81.6(\mathrm{SD}=9.5), 68.4 \%$ are females and $68.4 \%$ live alone. Measures: Socio-demographic data survey, Portuguese version of World Health Organization Quality of Life Instrument-Older Adults and an audiological evaluation constituted by an otoscopy, a tympanogram, a pure tone audiogram and the speech in noise test. Statistical analysis was performed using software IBM SPSS version 24. Nonparametric test were used.

Results:

When the elderly live alone, results showed significant positive associations between CAP and QoL (total score). There was positive correlations between QoL and the speech in noise test in the relation signal/noise $10 \mathrm{~dB}(\mathrm{r}=0.573 ; \mathrm{p}=0.041)$, and total $(\mathrm{r}=0.573 ; \mathrm{p}=0.040)$ for right ear. The same result was observed in the left ear when the relation signal/noise $10 \mathrm{~dB}(\mathrm{r}=0.572$; $\mathrm{p}=0.041)$, and total $(\mathrm{r}=0.583 ; \mathrm{p}=0.037)$. No significant results were obtained when the seniors don't live alone.

\section{Conclusion:}

The results evidenced the importance of sociodemographic factors, such as "live alone" in the relation between CAP and QoL in older adults. Probably when we live alone the discrimination of speaking in noisy ambiance is a predictor factor of quality of life in seniors. Future studies are needed in order to clarify this relation. 